\title{
The reconstruction after pylorus preserving pancreatoduodenectomy: pancreatogastrostomy with Roux-en-Y reconstruction versus conventional Traverso type reconstruction
}

\author{
Joji Yamamoto, Yoshiaki Shimizu, Motoki Nagai, Harufumi Makino, Shigehiro Kojima, Yuuki Ozamoto
}

Department of Surgery, Kamagaya General Hospital, Chiba, Japan

Received 16 October 2009; revised 11 January 2010; accepted 15 January 2010.

\begin{abstract}
Objective: To compare the result of pancreatogastrostomy (PG) with Roux-en-Y reconstruction versus pancreatojejunostomy (PJ) with conventional Traverso-type reconstruction following pylorus-preserving pancreatectomy (PP. PD) in a retrospective study. PPPD has been accepted as a radical surgical treatment for malignant periampullary neoplasms. However, the reconstruction of this surgery is technically complicated. The leakage of pancreatic juice and the delayed gastric emptying are the major complications of PPPD and may be fatal. To solve these problems, we have performed PG with Roux-en-Y anastomosis. There are several techniques proposed the reconstruction after PPPD, however there have been no previous study describing the result of PG with Rouxen-Y anastomosis. Since 2002 we have performed 32 cases of PPPD. They were divided into two groups according to the reconstruction procedures: PG with Roux-en-Y reconstruction (group PG-RY) (17 cases) and pancreatojejunostomy with Traverso-type reconstruction (group PJ-T) (15 cases). Results: Patient age, gender, and underlying disease were comparable among the groups. Two groups showed no differences in intraoperative bleeding amount, and the time of surgery. The occurrence of the pancreatic leakage was significantly reduced in the group PG-RY compared with the group PJ-T. There was no case of the delayed gastric emptying in the group PGRY. Conclusions: Pancreatogastrosotmy with Roux-en-Y anastomosis can reduce the occurrence of the pancreatic leakage and delayed gastric emptying following pylorus-preserving pancreatoduodenectomy.
\end{abstract}

Keywords: Pancreatoduodenectomy; Pancreatogastrostomy; Roux-en-Y Reconstruction; Pancreatojejunostomy; Traverso-Type Reconstruction

\section{INTRODUCTION}

Since Traverso and Longmire reported the validity of pylorus-preserving pancreatoduodenectomy (PPPD) for benign pariampullary disease in 1978 , this procedure has been practiced and accepted as a radical treatment for periampullary neoplasm. The 1999 National Survey of Pancreatic Cancer in Japan showed that PPPDs were performed in $42.6 \%$ of the periampullary cancers [1]. Recent advantages in surgical technique has reduced operative mortality after this procedure, however, the rate of post-operative complications is still high. The most frequent and severe complication is the leakage of pancreatic juice. The delayed gastric emptying (DGE) is a unique and frequent complication of PPPD. In some previous reports, the causes of this complication were speculated to be either the interruption of gastrointestinal continuity, angulation of the gastrointestinal tract, hormone imbalance or abnormal gastric peristalsis [2].

In an attempt to reduce the frequency and severity of post-operative complications in patients undergoing PPPD, we performed PG with Roux-en-Y reconstruction for the reconstruction. To date, we have performed this procedure in 17 cases of periampullary neoplasms and have not yet encountered fatal complications.

\section{PATIENTS AND METHODS}

Since 2002, thirty two cases of PPPD were performed for radical operations of periampullary neoplasms in our institute. These surgical procedures were carried out by 4 surgeons with sufficient experience. Out of these $32 \mathrm{pa}$ tients, 22 were male $(67.8 \%)$ and 10 were female $(31.2 \%)$ with a median age of $66.0 \pm 8.6$ years (range $46-82$ years). 
We divided these patients into two groups according to the reconstruction procedures: 17 cases of PG-RY, 15 cases of PJ-T. Clinical factors of these patients were shown in the Table 1. We analyzed the postoperative course in these 32 patients and compared the results between these two groups.

Delayed gastric emptying is defined as the nasogastric tube placement for more than 10 days postoperatively or the output of gastric aspiration from gastrostomy tube more than $200 \mathrm{ml}$. Pancreatic leakage is defined as an external discharge greater than $50 \mathrm{ml}$ obtained through drain or percutaneous aspiration, containing at least three times normal serum value of amylase, as described by Yeo et al. [3]. Major leakage of pancreatic juice was defined as a leakage with peripancreatic abscess formation. Biliary fistula was diagnosed by the distinctive color of discharge containing bilirubin. Postoperative mortality was defined as death occurring in the first 30 postoperative days or before discharge from the hospital.

\section{SURGICAL TECHNIQUE}

In PG with Roux-en-Y reconstruction, surgical procedures are following:

Pancreatogastrostomy: After all standard resections are completed, pancreatogastrostomy (PG) is performed. The cut end of the pancreas is reapproximated and closed for hemostasis. A short stenting tube is placed in the pancreatic duct and secured loosely with one absorbable surgical suture. A horizontal incision is made in the posterior wall of the stomach, followed by the invagination of the pancreatic remnant. A vertical incision is then made in the anterior wall of the stomach. Interrupted sutures are placed between the full thickness of the gastric wall and the pancreas parenchyma through the interior of the stomach. This anastomosis is secured with approximately 16 absorbable surgical sutures. A stenting tube is introduced into the stomach, which allows for free drainage of the pancreatic juices into the stomach. A gastrostomy tube is inserted into the stomach to reduce the internal pressure of the stomach.

Hepaticojejunostomy: Approximately $30 \mathrm{~cm}$ of the jejunum is separated with its pedicle. The jejunal limb is approximated and sutured to the proximal hepatic duct. A stenting tube is not inserted when the hepatic duct is dilated over $1 \mathrm{~cm}$ in diameter to reduce the incidence of retrograde infection. A continuous running suture using one absorbable suture line achieves speed. A drainage tube is not placed beside this anastomosis.

Duodenojejunostomy: An end-to-end anastomosis is made between the remnant jejunum and the pylorus.

Jejunojejunostomy: An end-to-end anastomosis is made between the separated and the remnant jejunum. The remnant jejunum is brought up on the anterior side of the transverse colon, so that we can avoid bowel stenosis in case of intraabdominal recurrence. The right gastric artery is not preserved in our method. The position of this anastomosis is approximately $20 \mathrm{~cm}$ downstream from the duodenojejunostomy. The importance of this reconstruction is to avoid the kink or torsion of the stomach and the anastomosed jejunum. This may help the transport of digestive contents (Figure 1(a)).

In Traverso-type reconstruction, the end-to-side pancreatojejunostomy was performed in two layers: a pan-

Table 1. Clinical factors in patients who underwent pylorus-preserving pancreatoduodenectomy.

\begin{tabular}{|c|c|c|c|c|}
\hline & & PG-RY & PJ-T & \\
\hline patients & & 17 & 15 & \\
\hline gender (male/female) & & 116 & 105 & NS \\
\hline \multirow{7}{*}{$\begin{array}{c}\text { age (years) } \\
\text { disease }\end{array}$} & & $70.1 \pm 8.0$ & $62.5 \pm 8.4$ & $\mathrm{p}=0.013$ \\
\hline & & & & \\
\hline & pancreas cancer & $9(52.9 \%)$ & $7(46.7 \%)$ & \\
\hline & bile duct cancer & $6(35.3 \%)$ & $2(13.3 \%)$ & \\
\hline & duodenal cancer & $1(5.9 \%)$ & $3(20.0 \%)$ & \\
\hline & IPMT & $1(5.9 \%)$ & 0 & \\
\hline & Vater cancer & 0 & $3(20.0 \%)$ & \\
\hline preoperative jaundice * & & $13(76.5 \%)$ & $5(33.3 \%)$ & $\mathrm{p}=0.266$ \\
\hline
\end{tabular}

PG-RY: Pancreatogastrostomy with Roux-en-Y reconstruction; PJ-T: Pancreatojejunostomy with Traverso type reconstruction; IPMT : intrapancreatic papillary mucinous tumor; NS: not significant.

* All the patients with obstructive jaundice underwent biliary drainage before the surgery. 


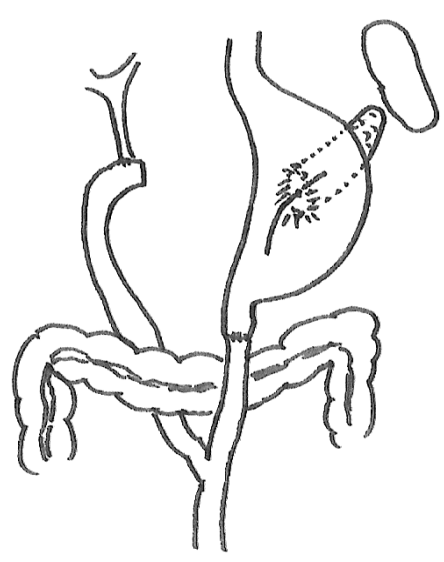

(a)

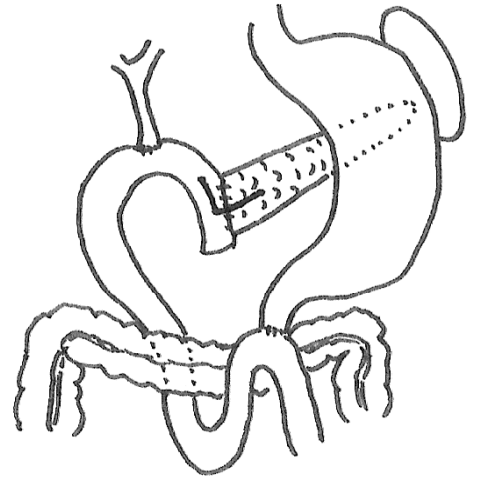

(b)

Figure 1. (a) Pancreatogastrostomy with Roux-en-Y reconstruction; (b) Pancreatojejunostomy with Traverso type reconstruction.

creatic duct and enteric mucosa interrupted suture and a pancreatic tissue to jejunal seromuscular layer using absorbable interrupted suture. Choledochojejunostomy was performed by the same technique introduced in Rouxen-Y anastomosis technique (Figure 1(b)).

Closed suction drain tubes were placed at the site of pancreatoenteric anastomosis in both procedures.

Statistical analysis:

Data are reported as mean \pm SEM. Statistical analysis was performed using the paired Student's t-test and Fisher's exact test for comparison between preoperative and postoperative status. Differences were considered as significant if $\mathrm{p}<0.05$.

\section{RESULTS}

Of the 32 patients analyzed, 17 patients received $P G$ with Roux-en-Y reconstruction (group PG-RY) and 15 patients received PJ with Traverso-type reconstruction. (group PJ$\mathrm{T})$. Operative and postoperative results were described in Table 2.

In PGRY group, there were 12 male and 5 female with mean age of $70.1 \pm 8.0$ years $(56-82)$. The average operative time was $430.3 \pm 71.0$ minutes. The average intraoperative bleeding amount was $988.2 \pm 498.9 \mathrm{ml}$.

In PJ goup, there were 10 male and 5 female with a mean age of $62.5 \pm 8.6$ years (46-78). The average operative time was $430.3 \pm 152.1$ minutes. The average intraoperative bleeding amount was $1076.0 \pm 670.9 \mathrm{ml}$.

Two groups showed no significant differences in gender, preoperative jaundice, the time of the surgery, and intraoperative bleeding amount.

As far as the pancreatic leakage is concerned, this complication rate is significantly reduced in PG-RY group compared with PJ-T group $(\mathrm{p}=0.015)$. There was no case of delayed gastric emptying in PG-RY group. $26.7 \%$ of the patients (4/15) in PJ-T group were suffered from DGE in our study. Two groups showed significant difference $(p=0.038)$. There was one case of mortality $(3.1 \%)$ in PJ-T group. The patient had liver cirrhosis as a preoperative complication, and died of liver failure 60 days postoperatively.

The postoperative complications in the patient with Roux-en Y with PG reconstruction were shown in Table 3.

\section{DISCUSSIONS}

Pancreatoduodenectomy (PD) has been considered as the only radical operative treatmetent for patients with malignant periampullary neoplasms. Recently pyloruspreserving PD (PPPD) is also accepted as a radical surgical treatment. However, the reconstruction after PPPD is technically complicated and the postoperative morbidity is still high. There are two major complications in this procedure, the one is the leakage of pancreatic juice and the other is the delayed gastric emptying.

The pancreatic leakage or fistula sometimes leads to fatal complications, including intraabdominal bleeding and sepsis. The rate of pancreatic leakage is reported to be $5 \%$ to $30 \%$ with a mortality rate of $6.5 \%$ to $8.4 \%$, and up to $50 \%$ of all death following pancreatoduodenectomy are results of the pancreatic anastomosis leakage [4]. Several techniques have been proposed for reducing the rate of pancreatic leakage and related complications. To avoid the risk of this occurrence, the administration of octreotide, which reduces the secretion of pancreatic juice, can be an option for preventing the leakage. However the use of octreotide is still controversial. Yeo etc. reported that the use of prophylactic octreotide does not reduce the incidence of pancreatic fistula after PD [5]. The pancreatic duct ligation without anastomosis or duct occlusion with fibrin glue can also be an option in select circumstances [6]. Several retrospective studies have reported low rates of pancreatic leakage and mortality 
Table 2. Operative and postoperative results.

\begin{tabular}{|c|c|c|c|c|}
\hline & & $\begin{array}{l}\text { PG-RY } \\
(\mathrm{n}=17)\end{array}$ & $\begin{array}{l}\text { PJ-T } \\
(n=15)\end{array}$ & \\
\hline Operation time (min.) & & $430 \pm 71.0$ & $430.3 \pm 152.1$ & NS \\
\hline Blood loss (ml) & & $988.2 \pm 498.9$ & $1076.0 \pm 670.9$ & NS \\
\hline Portal vein reconstruction & & $2(11.8 \%)$ & $3(20.0 \%)$ & NS \\
\hline \multicolumn{5}{|l|}{ Complications } \\
\hline & pancreatic leakage & $2(11.8 \%)$ & $5(33.3 \%)$ & $\mathrm{p}=0.015$ \\
\hline & delayed gastric emptying & 0 & $4(26.7 \%)$ & $\mathrm{p}=0.038$ \\
\hline & biliary leakage & $1(5.9 \%)$ & 0 & NS \\
\hline & liver failure & 0 & $1(6.7 \%)$ & NS \\
\hline Mortality & & 0 & $1(6.7 \%)$ & NS \\
\hline
\end{tabular}

NS: not significant.

Table 3. Postoperative complication in the patient with Roux-en $\mathrm{Y}$ with $\mathrm{PG}$ reconstruction.

\begin{tabular}{lc}
\hline \multicolumn{1}{c}{ Complication } & \\
\hline Surgical wound infection & 3 \\
Pneumonia & 1 \\
Small bowel obsrtuction & 1 \\
Pancreatic leakage & 2 \\
Reflux esophagitis & 1 \\
Bileperitoneum & 0 \\
Delayed gastric emptying & 0 \\
Death & None \\
\hline
\end{tabular}

after performing PG [7]. This procedure has several advantages over pancreatojejunostomy. Delcore et al. described several theoretic physiologic and technical advantages of performing pancreatogastrostomy [8]. First, the activation of the proteolytic enzymes can be avoided by this anastomosis. Enterokinase, which converts trypsinogen to its activated form trypsin, is present in small intestinal mucosa and it does not exist in gastric mucosa. The activation of trypin also depends on the presence of a neutral $\mathrm{pH}$. Second, the anatomical relationship between the remnant pancreas and the stomach is the advantageous for a tension-free anastomosis. The pancreas lies adjacent to the posterior gastric wall. At our institute, we invaginate the remnant pancreas into the stomach, and suture the pancreatic parenchyma and the full thickness of gastric wall inside of the stomach [9]. This is a simple and safe method for pancreatogastric anastomosis. A short stenting tube is used as a pancreatic duct that spontaneously falls out within a few months. We do not employ the long external drainage tube, because it often becomes twisted which produces high internal pressures within the pancreatic duct. In our 17 cases of PG-RY, 2 cases showed minor leakage of pancreatic juices, however, both cases resolved only by using drainage and discontinuation of oral feeding within 2 weeks. We have never used octreotide prophylactically in any patient.

The delayed gastric emptying (DGE) is a unique complication after PPPD. DGE is defined as the output of gastric aspiration from nasogastric tube or gastrostomy tube more than $200 \mathrm{ml}$. PPPD has a risk of $37.5 \%$ to $50 \%$ for developing DGE in the early postoperative course [10]. There is a report describing a higher rate of DGE with PG [11]. The causes of DGE are not clear and might be multifactorial. The low concentration of plasma motilin, leakage, cholangitis or bowel edema, are major factors contributing to the development of this complication [2]. Furthermore, recent studies show that a strong angulation or torsion at the site of duodenojejunostomy plays a role for this unique complication. To address this issue we compared PG with Roux-en-Y anastomosis to PJ with conventional Traverso-type reconstruction. Rouxen-Y anastomosis is a means by which this problem may be avoided. By implementing PG-RY procedure, we can prevent a strong angle and torsion between the pylorus and the jejunum. A gastrostomy tube placed for the reduction of the internal gastric pressure can also straighten the angle of this anastomosis. Our results suggest the advantage of PG-RY regarding the smoothness of gastric passage.

It is also important to avoid intraabdominal infection. The infection in the abdominal cavity results in the decrease in peristalsis of digestive tract movement. When constructing the anastomosis, care must be taken to re- 
duce the incidence of postoperative complication. Usually we place a closed suction drain tube only at the site of pancreatogastric anastomosis. In pancreatic surgery, multiple suction catheters are placed in relation to the biliary and pancreatic anastomoses. However, in doing this we must realize that drainage tubes are associated with the reflux infection in the abdominal cavity [12]. Therefore, at our institution we employ the use of few drainage and stenting tubes.

There is no agreement as to the best reconstruction method after PPPD [13]. With our experience, PG with Roux-en-Y reconstruction can reduce the rate of these two major complications as least as possible.

\section{REFERENCES}

[1] National survey of pancreatic cancer in Japan 1999 (written in Japanese) (2001) Pancreas, 16, 115-147.

[2] Takahata, S., Ohtsuka, T., Nabae, T., et al. (2002) Comparison of recovery of gastric Phase III motility and gastric juice output after different types of gastrointestinal reconstruction following pylorus-preserving pancreatoduodenectomy. Journal of Gastroenterol, 37, 596-603.

[3] Yeo, C.J., Cameron, J.L., Maher, M.M., et al. (1995) A prospective randomized trial of pancreaticogastrostomy versus pancreaticojejunostomy after pancreaticoduodenectomy. Annals of surgery, 222, 580-588.

[4] Fujino, Y., Suzuki, Y., Ajiki, T., et al. (2002) Risk factors influencing pancreatic leakage and the mortality after pancreaticoduodenectomy in a medium-volume hospital. Hepato-Gastroenterology, 49, 1124-1129.

[5] Yeo, C.J., Cameron, J.L., Lillemoe, K.D., et al. (2000) Does prophylactic octreotide decrease the rates of pan- creatic fistula and other complications after pancreaticoduodenectomy? Results of a prospective randomized placebo-controlled trial. Annals of surgery, 232, 419-429.

[6] Suc, B., Msika, S., Fingerhut, A., et al. (2003) Temporary fibrin glue occlusion of the main pancreatic duct in the prevention of intraabdominal complications after pancreatic resection. Annals of surgery, 237, 57-65.

[7] Fromm, D. and Schwarz, K. (2003) Ligation of the pancreatic duct during difficult operative circumstances. Journal of the American College of Surgery, 197, 943948.

[8] Delcore, R., Thomas, J.H., Pierce, G.E., et al. (1990). Pancreatogastrostomy: A safe drainage procedure after pancreatoduodenectomy. Surgery, 108, 641-645.

[9] Yamamoto, J., Shimamura, Y., Nakahara, H., et al. (1997) Simple technique for pancreatogastrostomy and the histological findings of pancreatogastric anastomosis. $\mathrm{Ar}$ chives of surgery, 132, 785-788.

[10] Pikarsky, A.J., Muggia-Sullum, M., Eid, A., et al. (1997) Pancreaticogastrostomy after pancreatoduodenectomy. Archives of surgery, 132, 296-299.

[11] Weller, U., Makowiec, F., Fischer, E., et al. (2009) Reduced postoperative pancreatic fistula rate after pancreatogastrostomy versus pancreaticojejunostomy. Journal of Gastrointestinal Surgery, 13, 745-751.

[12] Conlon, K.C., Labow, D., Leung, D., et al. (2001) Prospective randamized clinical trial of the value of intraperitoneal drainage after pancreatic resection. Archives of surgery, 234, 487-494.

[13] Duffas, J.P., Msika, S., Fourtanier, G., et al. (2005) A controlled multicenter trial of pancreatogastrostomy or pancreatojejunostomy after pancreatoduodenectomy. American Journal of Surgery, 189, 720-729. 